\title{
A Nanowire-Based Plasmonic Quantum Dot Laser
}

\author{
Jinfa Ho ${ }^{*}{ }^{1}$, Jun Tatebayashi ${ }^{1}$, Sylvain Sergent ${ }^{1}$, Chee Fai Fong ${ }^{2}$, Yasutomo Ota ${ }^{1}$, Satoshi \\ Iwamoto $^{1,2}$, and Yasuhiko Arakawa ${ }^{1,2}$ \\ ${ }^{1}$ Institute for Nano Quantum Information Electronics and ${ }^{2}$ Institute of Industrial Science, The \\ University of Tokyo, 4-6-1 Komaba, Meguro-ku, Tokyo 153-8505, Japan
}

\section{SUPPORTING INFORMATION}

\section{Comparison between fundamental plasmonic mode, higher order plasmonic mode, and plasmon-waveguide hybrid mode}

The main motivation for placing the nanowires in direct contact with silver is the improved mode overlap with the nanowire core region compared to the conventional plasmon-waveguide hybrid mode supported by nanowire plasmonic lasers separated from the metallic substrate by a thin low-index dielectric layer. Figure S1 shows the calculated real part of the effective indices, the propagation losses $\alpha_{\mathrm{p}}$, and confinement factors $\Gamma$ (defined as the ratio of the electric field confined in the nanowire core region containing the quantum dots to the total electric field) for the fundamental plasmonic mode, the higher order plasmonic mode, and the plasmon-waveguide hybrid mode. To support the plasmon-waveguide hybrid mode in the simulations, a $5 \mathrm{~nm}$ thick $\mathrm{SiO}_{2}(n=1.5)$ layer was inserted between the nanowire and the silver layer. Compared to the plasmon-waveguide hybrid mode, both the fundamental and higher order plasmonic modes 
supported by nanowires directly in contact with silver exhibit larger confinement factors. The normalized field distributions also show that the plasmon-waveguide hybrid mode is strongly confined in the low-index dielectric region and is expected to interact weakly with quantum dots that are embedded in the nanowire core. While the fundamental plasmonic mode has the best mode overlap with the quantum dot region, its propagation losses are too large to be overcome by the gain provided by the quantum dots. The higher order plasmonic mode on the other hand has significantly lower losses comparable to the plasmon-waveguide hybrid mode due to a large portion of the mode residing in the non-metallic regions. It also has a relatively good mode overlap with the quantum dots, and is thus the most promising for achieving lasing with our quantum dot in nanowire samples.

\section{Absence of lasing leaky photonic modes}

In addition to the plasmonic modes, leaky photonic modes are also possible lasing modes below the cut-off diameter of photonic modes ${ }^{1}$. Such leaky photonic modes can exist below the cut-off due to gain guiding effects, and leak energy to the surface plasmon polariton (SPP) mode of the silver-air interface. Near the cut-off of the photonic modes at a nanowire diameter of $210 \mathrm{~nm}$, there exists four modes: the fundamental and higher order plasmonic modes, as well as the $\mathrm{HE}_{11 \mathrm{a}}$ and $\mathrm{HE}_{11 \mathrm{~b}}$ photonic modes. The calculated mode spacings using Eq. 1 in the main text of these four modes are shown in Fig. S2a, along with the mode distributions of the HE photonic modes. Their effective mode indices and propagation losses are plotted in Fig. S2b and S2c, respectively. At larger nanowire diameters, the HE modes have photonic characteristics and minimal overlap with the silver layer. As the nanowire diameter gets smaller, the HE modes acquire strong 
plasmonic characteristics due to larger overlap with the silver layer. In particular, the $\mathrm{HE}_{11 \mathrm{~b}}$ mode distribution is quite similar to the fundamental plasmonic mode, while the $\mathrm{HE}_{11 \mathrm{a}}$ mode distribution is similar to the higher order plasmonic mode. The emission polarizations of the HE modes are also similar to the plasmonic modes they resemble. Based on just polarization measurements, we cannot identify if the lasing mode is the higher order plasmonic mode or the $\mathrm{HE}_{11 \mathrm{a}}$ leaky mode. The expected mode spacings however are quite different for these two modes at small nanowire diameters as shown in Fig. S2a. Based on the observed mode spacing, the lasing mode should originate from the higher order plasmonic mode rather than the HE $\mathrm{H}_{1 \mathrm{a}}$ leaky mode. The $\mathrm{HE}_{11 \mathrm{a}}$ mode profile (Fig. S2a) also shows poor overlap with the quantum dot region and it is unlikely for the $\mathrm{HE}_{11 \mathrm{a}}$ mode to be lasing.

The group index $n_{g}$ can be obtained by

$$
n_{g}=n_{e f f}-\lambda \frac{d n_{e f f}}{d \lambda}
$$

At large nanowire diameters $(d=400 \mathrm{~nm})$, the HE modes have small $n_{g}$ values of 4.1 and 4.4 for the $\mathrm{HE}_{11 \mathrm{a}}$ and $\mathrm{HE}_{11 \mathrm{~b}}$ modes, respectively. Near the cut-off of the HE modes $(d=210 \mathrm{~nm})$, the $\mathrm{d} n_{\text {eff }} / \mathrm{d} \lambda$ term increases considerably, resulting in large group indices of 7.5 and 8.2 for the $\mathrm{HE}_{11 \mathrm{a}}$ and $\mathrm{HE}_{11 \mathrm{~b}}$ modes, respectively. In contrast, $n_{g}$ at $d=210 \mathrm{~nm}$ for the fundamental and higher order plasmonic modes are 7.1 and 5.3, respectively. The large difference in $n_{g}$ allows for unambiguous distinction between the higher order plasmonic mode and the $\mathrm{HE}_{11 \mathrm{a}}$ mode near cutoff.

The lack of leaky photonic modes that can lase in our structure can be explained by the long operation wavelength. The emission of our samples is centered at $\sim 850 \mathrm{~nm}$, which is far from the surface plasma wavelength of $\sim 450 \mathrm{~nm}$ for a silver-air interface ${ }^{2}$, and SPP penetration into 
the silver layer is reduced. Using the silver permittivity values from ref 3, we calculate the propagation losses of the silver-air interface SPP mode to be $\sim 24 \mathrm{~cm}^{-1}$ and the effective index to be $\sim 1.01$, which is close to free space modes. The interface SPP mode loss is lower than that for the HE modes, which are in excess of $200 \mathrm{~cm}^{-1}$ near cut-off. The condition for leaky photonic modes that can lase requires that the interface SPP mode has larger effective indices and losses than the HE modes ${ }^{1}$. At $850 \mathrm{~nm}$, this condition is not met and the leaky photonic modes have vanishingly small confinement factors. The presence of lasing leaky photonic modes in our lasing samples with diameters of $\sim 180 \mathrm{~nm}$ can therefore be eliminated.

\section{FDTD simulation of near-field radiation pattern}

To verify the different polarizations observed for the fundamental and higher order plasmonic modes, finite-difference time-domain (FDTD) simulations were performed using Lumerical FDTD Solutions 8.12. We used refractive indices of 3.66 for GaAs, 3.62 for $\mathrm{AlGaAs}{ }^{4}$, and 3.38

for $\operatorname{In}_{0.22} \mathrm{Ga}_{0.78} \mathrm{As}{ }^{5}$ in order to replicate the nanowire structure as shown in Fig. 1a of the main text. The material properties of silver were obtained from ref 3 . In these simulations, the nanowire length is set to $2 \mu \mathrm{m}$ while the diameters are $180 \mathrm{~nm}$ for nanowires that support the higher order plasmonic mode and $150 \mathrm{~nm}$ for nanowires that support only the fundamental plasmonic mode. The modes are launched from the middle of the nanowires in the $+z$-direction, and due to propagation losses the scattered field from the top facet is stronger than the scattered field from the bottom facet. Figure S3a shows the normalized near-field emission patterns, resolved in the directions parallel and perpendicular to the nanowire long axis. Due to the significant difference in intensities of the scattered fields from each facet, interference patterns 
cannot be observed in the images. The simulation results are thus similar to the microscope image for the fundamental plasmonic mode shown in Fig. $3 \mathrm{~b}$ of the main text. The relative field intensities in the directions parallel and perpendicular to the nanowire long axis are consistent with the measured polarization dependences for each mode. The interference pattern in the lasing sample can be reproduced by performing simulations with two coherent dipole emitters placed at each facet, as shown in Fig. S3b, which is in good agreement with the microscope image of the nanowire emission obtained experimentally.

\section{Longitudinal mode distribution}

The longitudinal mode distribution is shown in Fig. S4. The FWHM of the antinodes is $\sim 180 \mathrm{~nm}$, and the distance between the nodes are $\sim 360 \mathrm{~nm}$. For regularly spaced quantum dots that have a period that is smaller than the distance between the nodes, we estimate that $\sim 50 \%$ of the dots can interact with the higher order plasmonic mode.

\section{Rate equation fitting}

The laser output power and linewidth were obtained by fitting the power-dependent photoluminescence spectra with three Gaussian curves. The background spontaneous emission is

fitted by a broad Gaussian curve, while sharper Lorentzian peaks are used for the Fabry-Pérot modes. For the rate equation fitting, only the area under the lasing peaks were used. 
To estimate the gain and spontaneous emission coupling factors, we use a simple rate equation analysis based on refs 6 and 7 , given by

$$
\begin{gathered}
\frac{d n}{d t}=\sigma p(t)-A n-\zeta A s\left(n-n_{0}\right)-\Gamma_{n r} n \\
\frac{d s}{d t}=\beta A n+\zeta A s\left(n-n_{0}\right)-\gamma_{p h} S
\end{gathered}
$$

where $n$ is the carrier number, $s$ is the photon number and $n_{0}$ is the carrier number at transparency which we set to volume ${ }_{N W} \times 2.0 \times 10^{18} \mathrm{~cm}^{-3} 8 \cdot p(t)=p_{0} \exp \left(-\frac{\left(t-t_{0}\right)^{2}}{\Delta t^{2}}\right)$ is the incident pulsed pump laser and we define the pulse width $\Delta t$ to be 50 ps. $\sigma$ is the pump laser photon to semiconductor carrier conversion efficiency. The carrier decay rate $A$ is set to $1 \mathrm{~ns}^{-18}$, and no Purcell factor modifications are applied as the quantum dots are located away from the region of strong electric field intensities for the higher order plasmonic mode. The mode overlap with the gain medium $\zeta \sim 0.07$ was obtained from finite difference eigenmode simulations (see Fig. S1). The photon decay rate $\gamma_{p h}$ in the cavity can be calculated by $\gamma_{p h}=\alpha_{T} v$ where $v$ is the group velocity of the mode and total $\operatorname{loss} \alpha_{T}=\alpha-\frac{1}{L} \ln (R)$ for a Fabry-Pérot cavity. Based on the reflectance $R$ of $\sim 0.8$ obtained from FDTD simulations, $\gamma_{p h}$ is $\sim 0.012 \mathrm{fs}^{-1}$. It should be noted however that the reflectance is likely to vary from sample to sample due to the ultrasonication breaking process, which results in imperfectly cleaved bottom facets. The spontaneous emission coupling factor $\beta$ was obtained from rate equation fitting. As an example, the fitting for the nanowire with $l \sim 2.3 \mu \mathrm{m}$ and $w \sim 180 \mathrm{~nm}$ at $7 \mathrm{~K}$ is shown in Fig. S5a. A $\beta$ of 0.005 gave the best fit to the experimental data. Finally, the non-radiative recombination rate $\Gamma_{n}$ is assumed to be negligible in the rate equation fitting. The effect of increasing $\Gamma_{n r}$ is to result in 
a more pronounced non-linear kink at threshold and can be offset by a larger $\beta$. Therefore, the $\beta$ factors obtained in our fitting is the lower bound estimate. However, it can be seen in Fig. 4a of the main text that at higher temperature the non-linear kink becomes more pronounced, and this is likely due to the increased non-radiative recombination that was not taken into account in our rate equation.

The average gain per pulse can be calculated by

$$
g=\frac{\int \zeta A s\left(n-n_{0}\right) d t}{v \int s d t}
$$

This is shown in Fig. S5b and saturates at $2400 \mathrm{~cm}^{-1}$, consistent with the calculated cavity losses. After accounting for the fact that only $50 \%$ of the quantum dots couple to the lasing mode, the total gain required is $\sim 4800 \mathrm{~cm}^{-1}$. It should be noted that the loss calculations were based on optical properties of silver measured at room temperature ${ }^{3}$, and at cryogenic temperatures electron collision with the silver lattice atoms can be significantly reduced, leading to smaller propagation losses. In addition, the large density of states available in the quantum dot excited states can contribute to the gain required for achieving lasing ${ }^{10}$.

\section{Evidence for quantum dot emission}

To confirm that the observed emission in our samples originates from the quantum dot structures rather than quantum well or bulk materials, we demonstrate here single photon emission from nanowires containing a single quantum dot. Figure S6(a) shows the emission spectrum of one such sample at $14 \mathrm{~K}$, excited under $\mathrm{CW}$ conditions with a $800 \mathrm{~nm}$ laser at $100 \mathrm{nW}$. The emission 
from the higher intensity peak at $867 \mathrm{~nm}$ was selectively filtered and directed to a HanburyBrown and Twiss (HBT) setup consisting of two avalanche photodiodes (APDs) and a 50/50 beam splitter. The relative delay between the incidence of photons on the two APDs is measured and plotted as a histogram in Figure S6(b). This measurement is proportional to the second order correlation function given by:

$$
g^{(2)}(\tau)=\frac{\langle I(t) \cdot I(t+\tau)\rangle}{\langle I(t)\rangle^{2}}
$$

where $I(t)$ is the intensity of the emitted light at time $t$. A $g^{(2)}(0)$ of less than 0.5 indicates the detection of a single photon from a single quantum dot. As seen in Figure S6(b), the suppressed coincidence count at $t=0$ is direct evidence of single photon emission originating from a quantum dot in our sample.

In addition, we also demonstrate here the tunability of the emission peak as a function of the quantum dot thickness. Figure S7(a) shows the $7 \mathrm{~K}$ photoluminescence spectra of as-grown GaAs nanowire samples containing $\operatorname{In}_{0.22} \mathrm{Ga}_{0.78} \mathrm{As}$ quantum dots, with various quantum dot heights tuned by varying their growth duration. A redshift is observed with increasing quantum dot thickness due to a reduction in quantum confinement along the vertical direction. Figure S7(b) shows the calculated results of the electron-heavy hole transition wavelength of the quantum dots by a single-band effective mass approximation via self-consistent PoissonSchrödinger calculations. The transition wavelengths of $\operatorname{In}_{0.22} \mathrm{Ga}_{0.78} \mathrm{As}$ quantum dots formed on the (100) plane, (111)B plane with strain, and (111)B plane without strain were calculated as a function of the quantum dot thickness. The calculated emission wavelengths for quantum dots formed on the (100) plane are consistent with the experimentally observed emission peak wavelengths for smaller quantum dot thicknesses $(<12 \mathrm{~nm})$. Quantum dots with larger 
thicknesses show a closer agreement to that grown on the (111)B plane without strain, implying strain relaxation in the lateral direction. The results shown here enable the correlation of the quantum dot height with the emission wavelength of the quantum dots. 


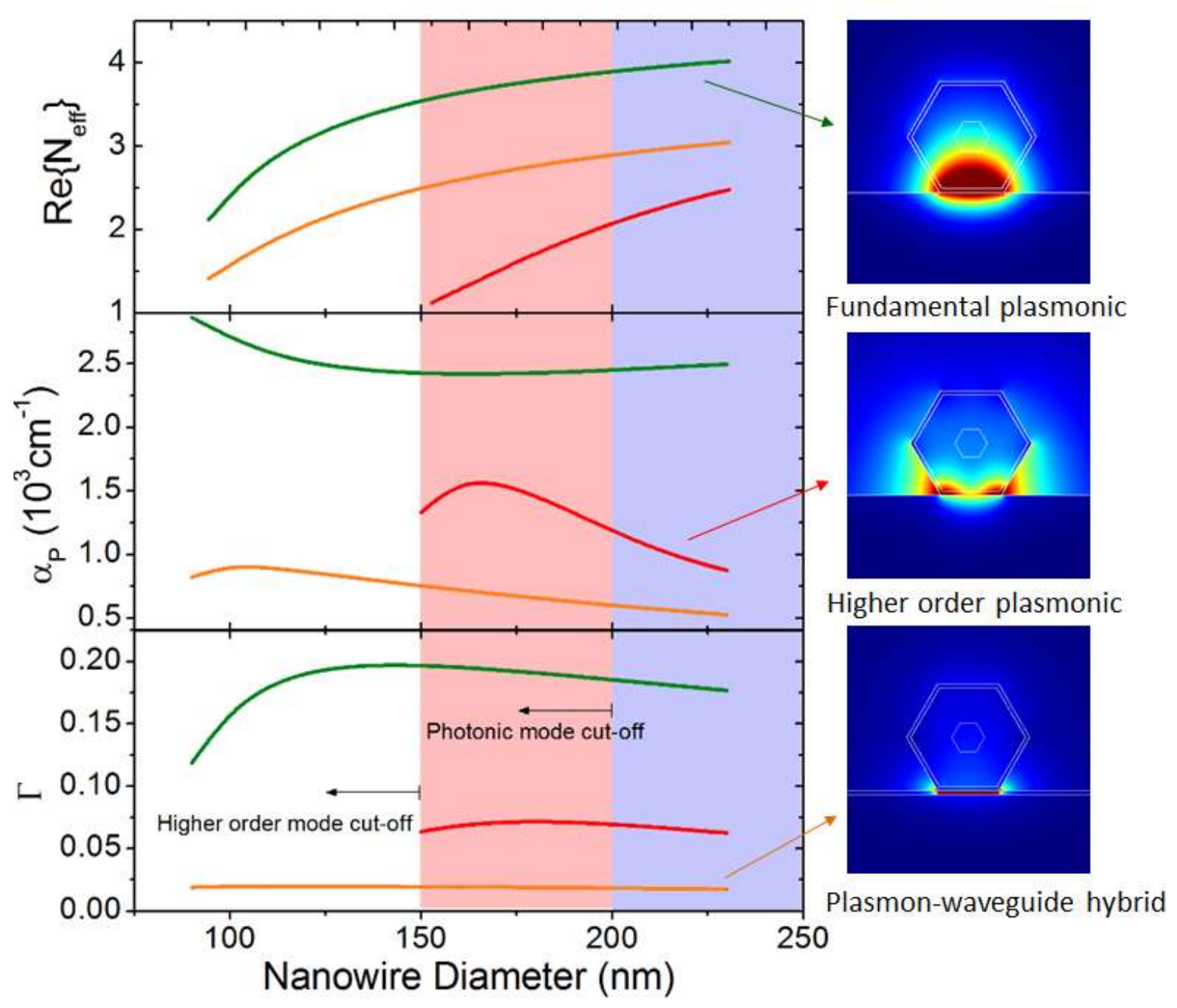

Figure S1. Calculated real part of the effective indices, propagation losses $\alpha_{p}$, and confinement factors $\Gamma$ (defined as the fraction of the electric field residing in the quantum dot region shown as the innermost hexagon in the field distribution images) of the fundamental plasmonic mode (green curves), the higher order plasmonic mode (red curves), and the plasmon-waveguide hybrid mode (orange curves), as a function of the nanowire diameter. Insets show the normalized field distributions for the fundamental plasmonic mode (top), the higher order plasmonic mode (middle) and the plasmon-waveguide hybrid mode (bottom). The higher order plasmonic mode 
requires a relatively large contact area between the nanowire and the silver film, and is cut-off for nanowires with diameters below $\sim 150 \mathrm{~nm}$ for an operational wavelength of $850 \mathrm{~nm}$.

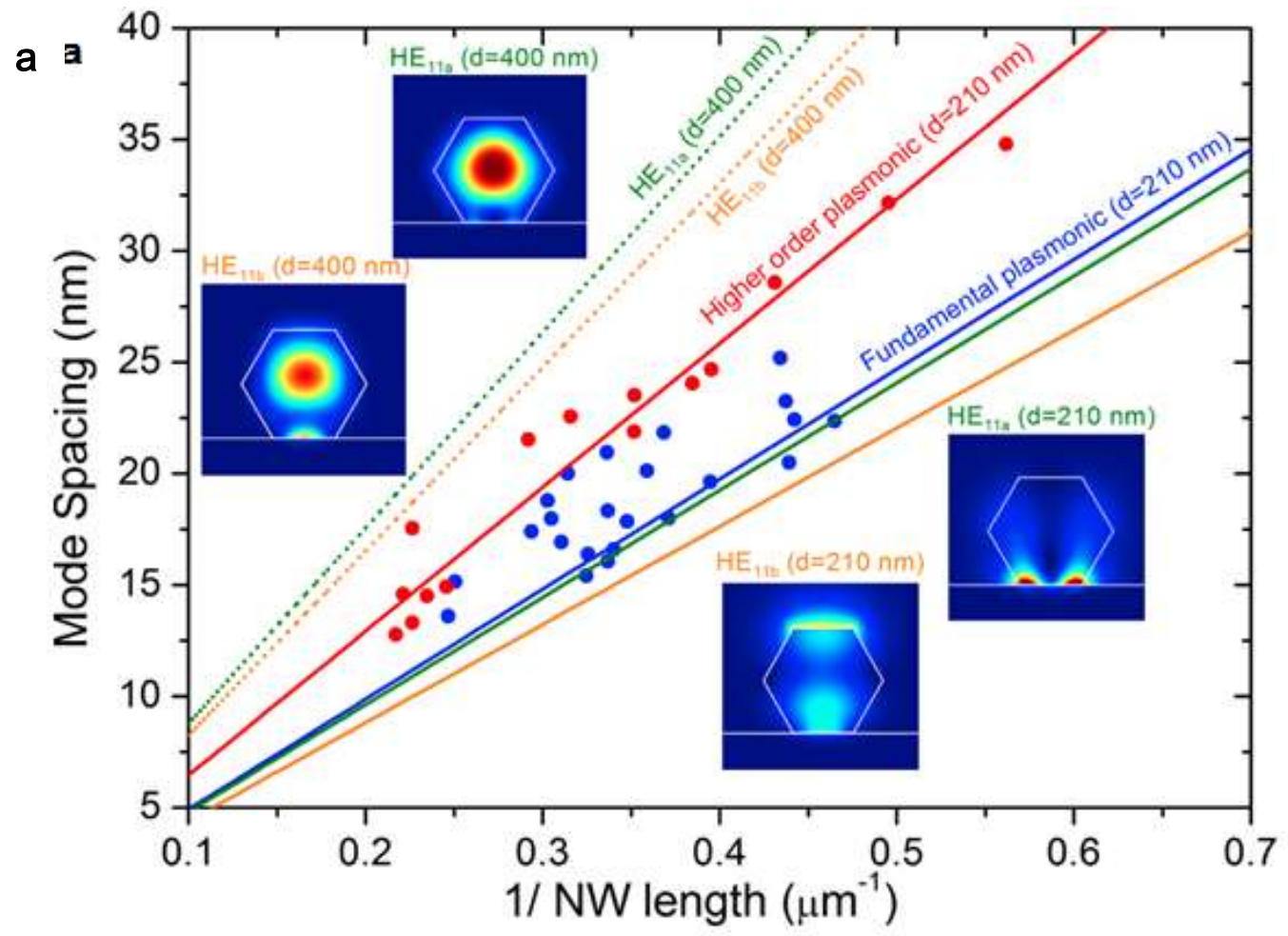

b

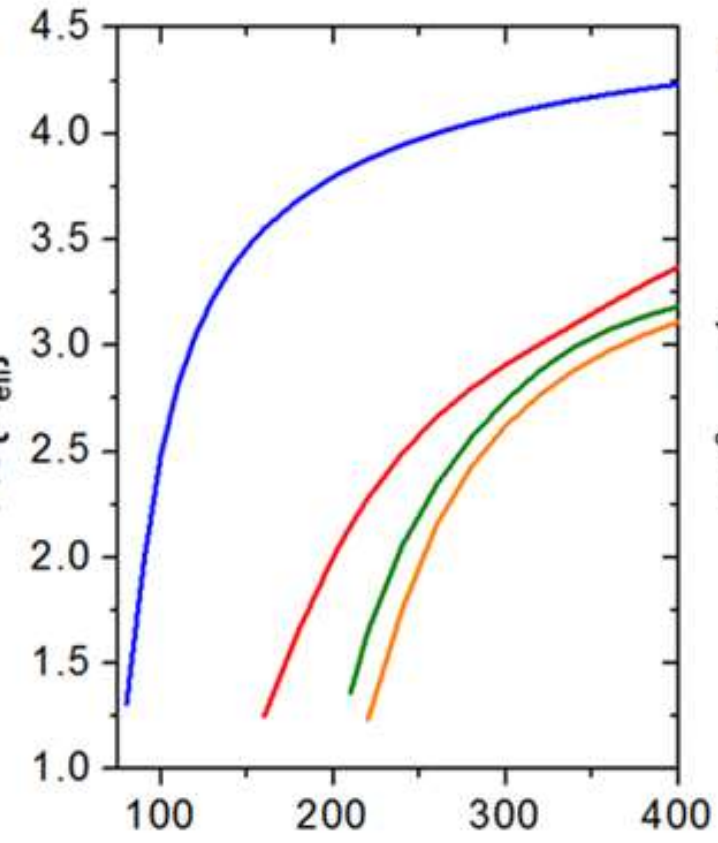

Nanowire Diameter $(\mathrm{nm})$

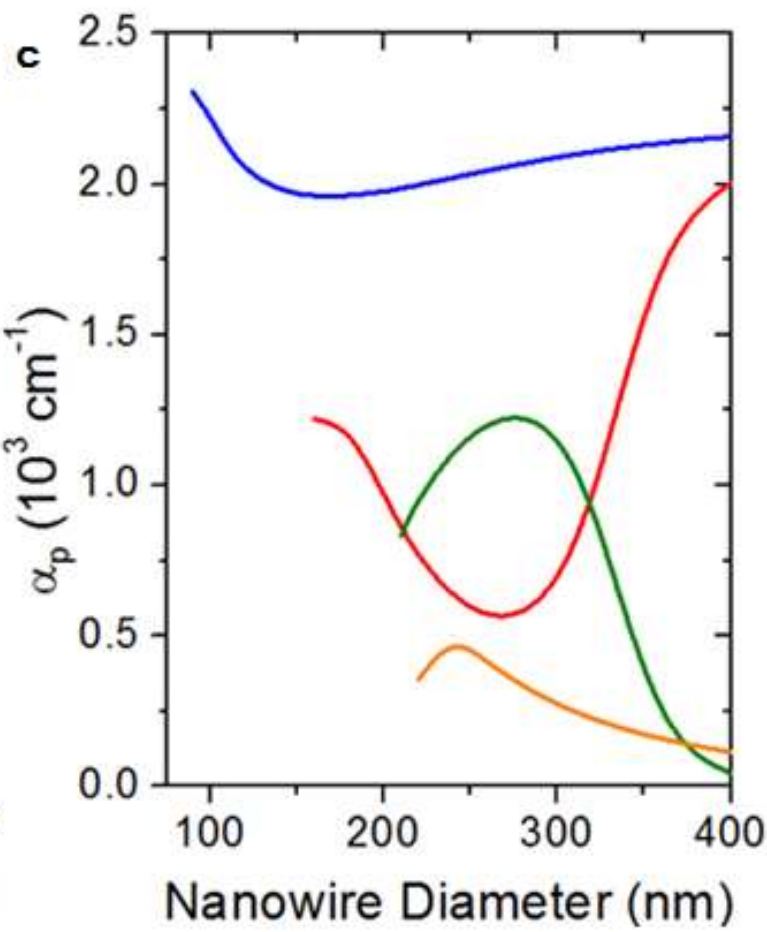


Figure S2. (a) Calculated mode spacings using equation (1) in the main text for the fundamental plasmonic mode (blue line), and higher order plasmonic mode (red line). The mode spacings for the $\mathrm{HE}_{11 \mathrm{a}}$ (green lines) and $\mathrm{HE}_{11 \mathrm{~b}}$ (orange lines) are shown for nanowire diameters of $400 \mathrm{~nm}$ (dotted lines) and $210 \mathrm{~nm}$ (solid lines). The insets show the field distributions for the $\mathrm{HE}_{11 \mathrm{a}}$ and $\mathrm{HE}_{11 \mathrm{~b}}$ modes at these two nanowire diameters. As the HE modes approach cut-off, their group indices increase considerably, exceeding that of the plasmonic modes. The large mode spacing difference between the higher order plasmonic mode and the $\mathrm{HE}_{11 \mathrm{a}}$ mode at small nanowire diameters allows for unambiguous distinction between the two modes. (b) Real part of the effective indices and (c) propagation losses for the fundamental plasmonic mode (blue line), higher order plasmonic mode (red line), $\mathrm{HE}_{11 \mathrm{a}}$ mode (green line) and $\mathrm{HE}_{11 \mathrm{~b}}$ mode (orange line). The HE photonic modes are cut-off below diameters of $200 \mathrm{~nm}$. Due to considerable overlap between the HE photonic modes and the silver layer at small nanowire diameters, their losses increase greatly. In comparison, the silver-air interface SPP has a propagation loss of $\sim 24 \mathrm{~cm}^{-1}$, which is much smaller than the losses of the HE modes near their cut-off. 
a

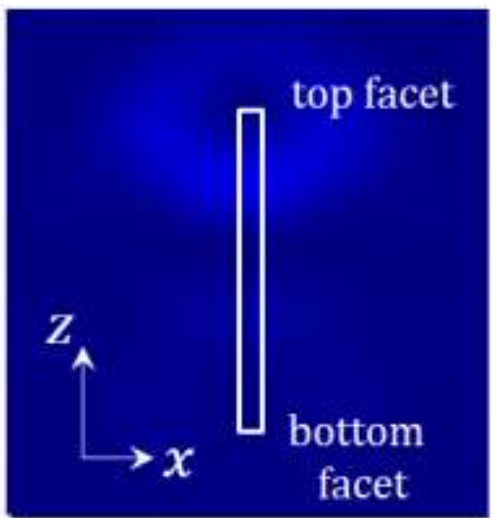

$\left|E_{x}\right|^{2}$ Fundamental

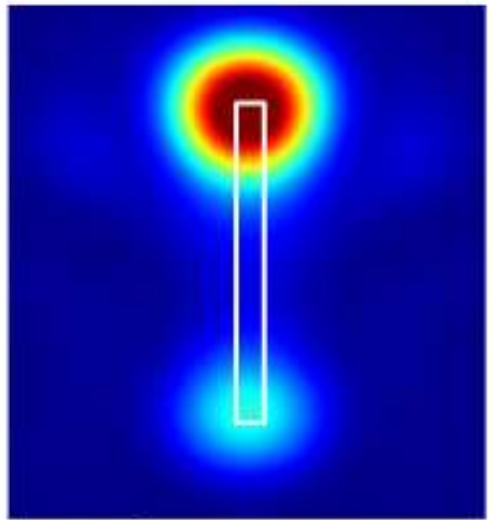

$\left|E_{x}\right|^{2}$ Higher order

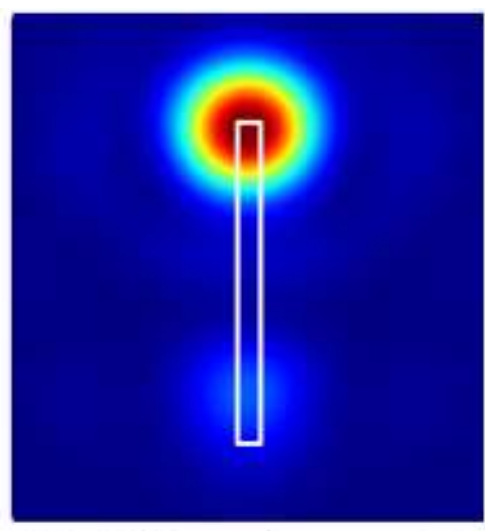

$\left|E_{z}\right|^{2}$ Fundamental

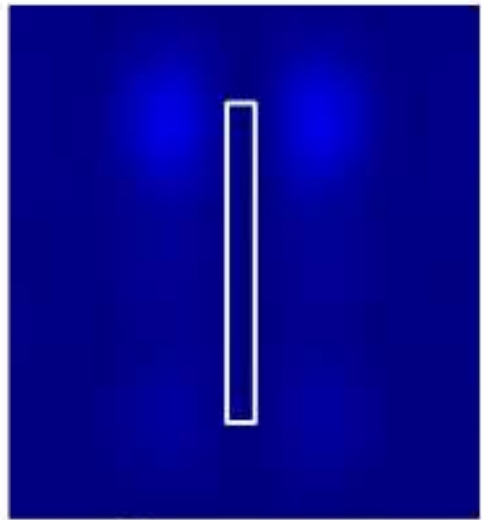

$\left|E_{z}\right|^{2}$ Higher order b
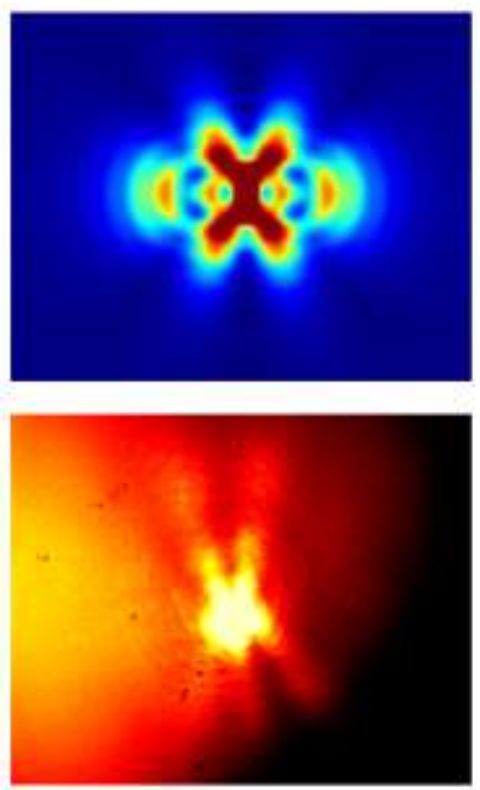

Figure S3. (a) Normalized near-field emission patterns for the fundamental and higher order plasmonic modes, resolved in the directions parallel to the nanowire axis ( $z$-direction) and perpendicular to the nanowire axis ( $x$-direction). The fundamental mode is polarized in the $z$ direction, while the higher order mode is polarized in the $x$-direction. (b) Near-field radiation pattern obtained with two coherent dipoles placed $2 \mu \mathrm{m}$ apart. The interference pattern is in good agreement with that of the lasing sample shown here for comparison. 


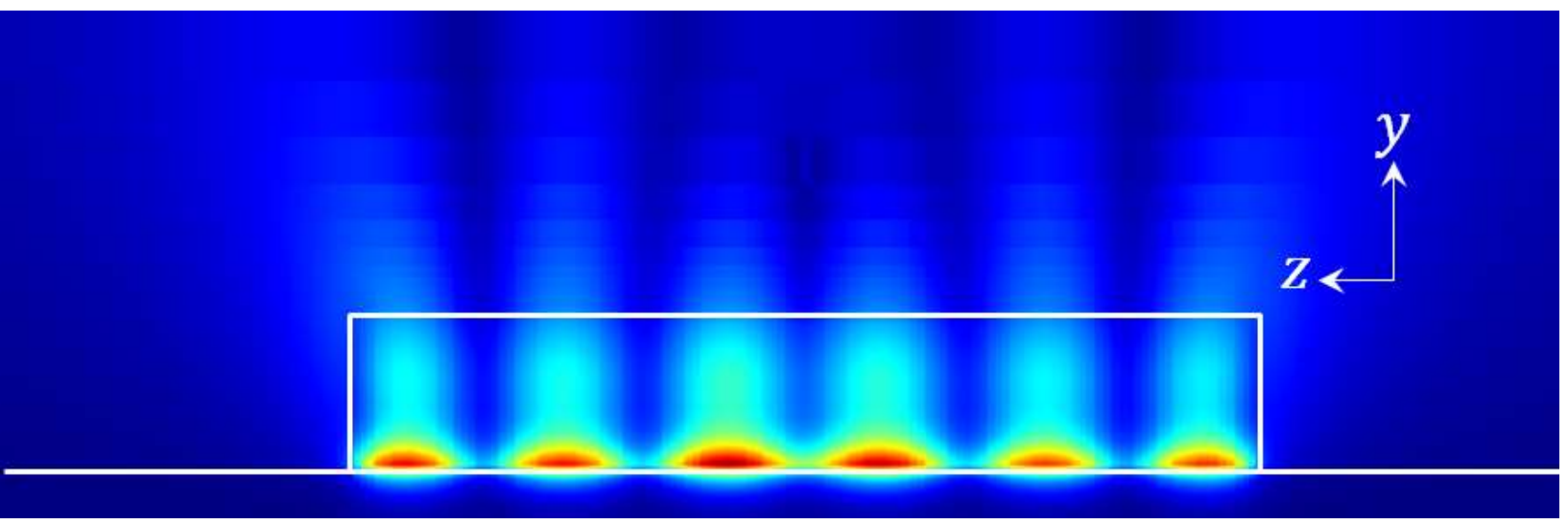

Figure S4. Longitudinal mode distribution of the electric field for the higher order plasmonic mode.
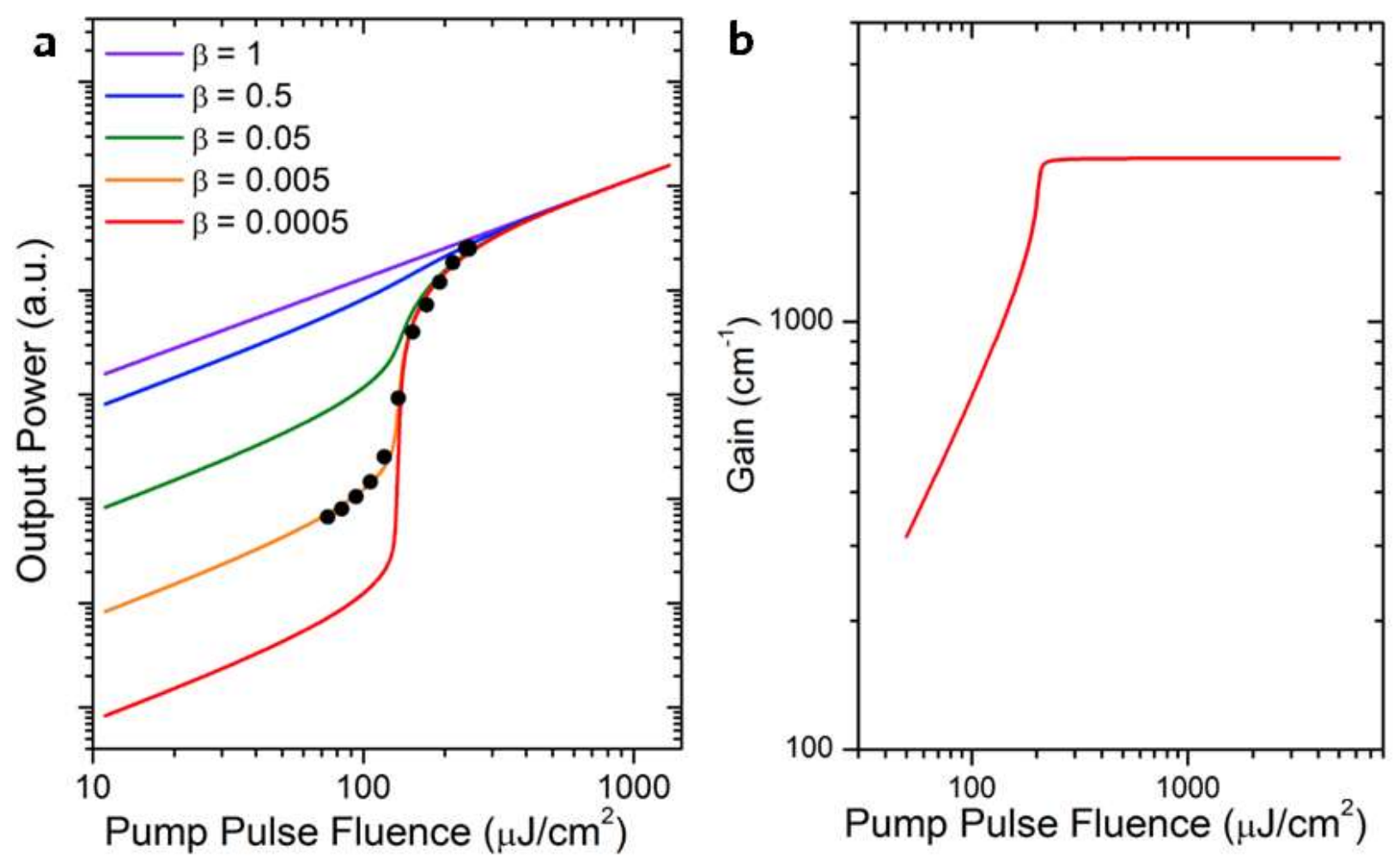

Figure S5. (a) Calculated output power with varying spontaneous emission $\beta$ factors, and the experimentally obtained output power (black circles) for a nanowire with $l \sim 2.3 \mu \mathrm{m}$ and $w \sim 180$ 
$\mathrm{nm}$. The best fit was obtained for a $\beta$ factor of 0.005 . (c) Calculated average gain per pump pulse, showing gain saturation above threshold at $\sim 2400 \mathrm{~cm}^{-1}$.

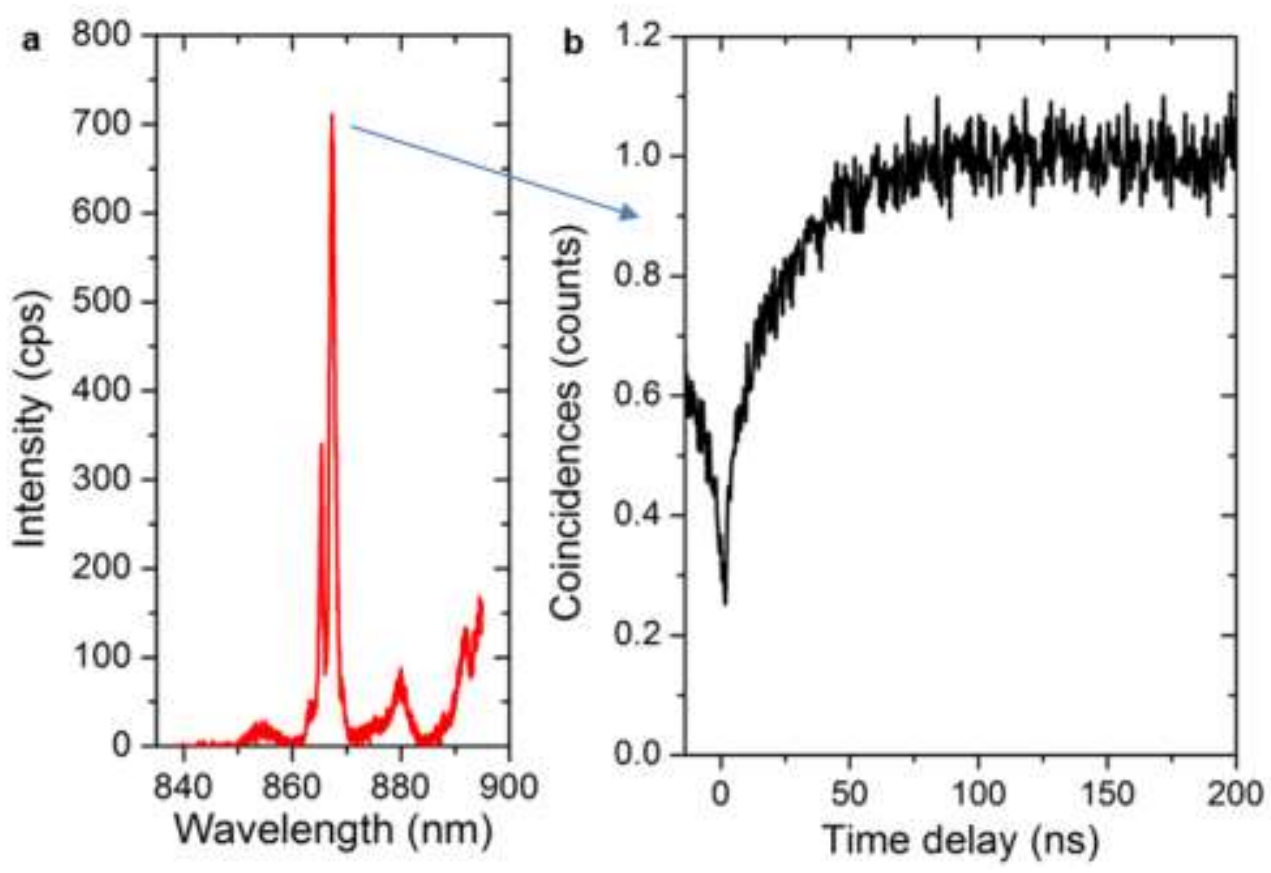

Figure S6. (a) $14 \mathrm{~K}$ PL spectrum of a nanowire containing a single quantum dot, excited under $\mathrm{CW}$ conditions at $100 \mathrm{nW}$. (b) Coincidence count histogram of the $867 \mathrm{~nm}$ peak, measured by a HBT setup. The $g^{(2)}(0)$ is $\sim 0.25$, which is clear evidence for single photon emission and that the $867 \mathrm{~nm}$ peak in (a) originates from a single quantum dot. 

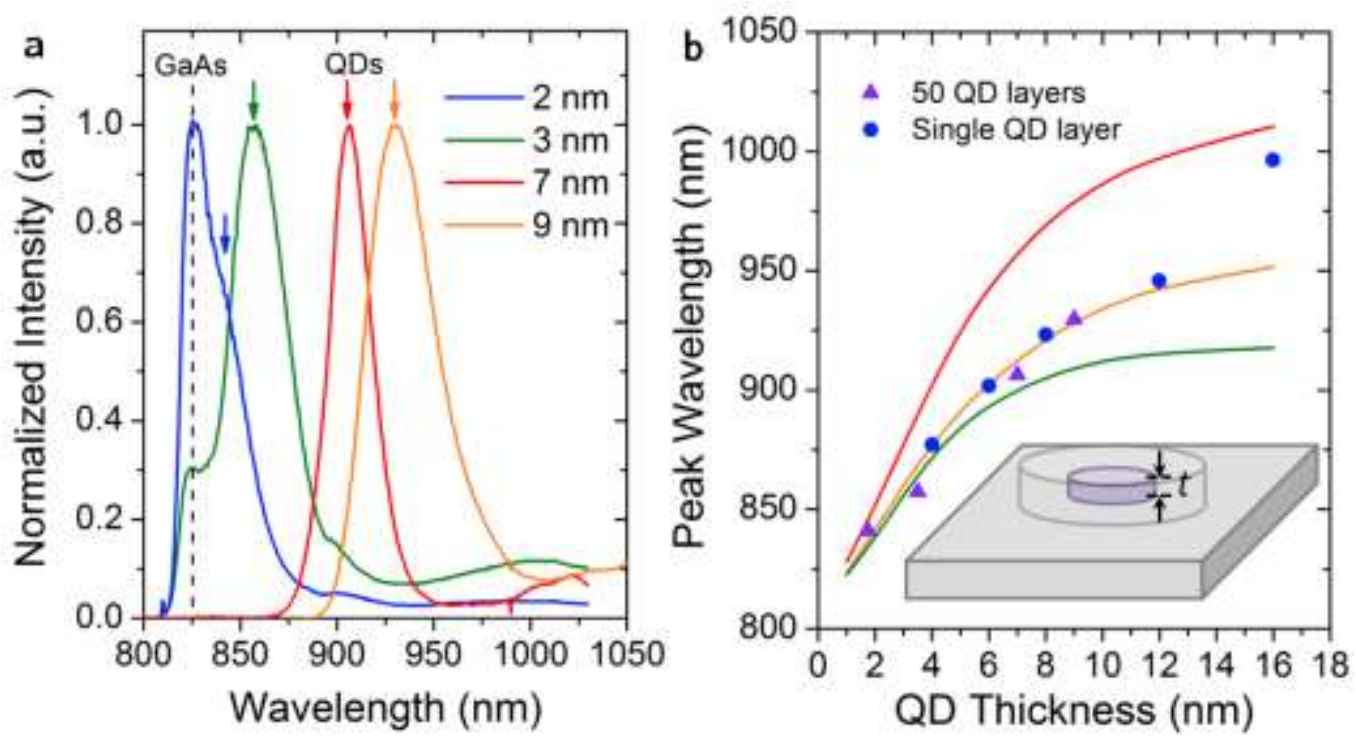

Figure S7. (a) PL spectra of as-grown nanowire samples with $50 \operatorname{In}_{0.22} \mathrm{Ga}_{0.78} \mathrm{As}$ QD layers with various QD thicknesses ranging from 2 to $9 \mathrm{~nm}$, at $7 \mathrm{~K}$ with an excitation power of $280 \mathrm{~mW} / \mathrm{cm}^{2}$. (b) Experimentally observed emission peak wavelengths for nanowires with 50 QD layers (purple triangles) and a single QD layer (blue circles) for comparison. The lines show the calculated electron to heavy hole transition energy by a single-band effective mass approximation of a cylindrical QD-in-nanowire structure (shown in inset), formed on the (100) plane (orange line), (111)B plane with strain (green line) and (111)B plane without strain (red line). 


\section{REFERENCES}

(1) Wuestner, S.; Hamm, J.M.; Pusch, A.; Hess, O. Plasmonic leaky-mode lasing in active semiconductor nanowires. Laser Photonic Rev. 2015, 9, 256.

(2) Maier, S.A. Plasmonics: Fundamentals and Applications, Springer Science+Business Media LLC, New York, 2007.

(3) Johnson, P.B.; Christy, R.W. Optical Constants of the Noble Metals. Phys. Rev. B 1972, 6, 4370.

(4) Aspenes, D.E.; Kelso, S.M.; Logan, R.A.; Bhat, R. Optical properties of $\mathrm{Al}_{\mathrm{x}} \mathrm{Ga}_{1-\mathrm{x}} \mathrm{As.} J$. Appl. Phys. 1986, 60, 754.

(5) Takagi, T. Refractive index of $\mathrm{Ga}_{1-\mathrm{x}} \mathrm{In}_{\mathrm{x}} \mathrm{As}$ Prepared by Vapor-Phase Epitaxy. Jap. J. Appl. Phys., 1978, 17, 1813.

(6) Yokoyama, H.; Brorson, S.D. Rate equation analysis of microcavity lasers. J. Appl. Phys. 1989, 66, 4801 .

(7) Oulton, R.F.; Sorger, V.J.; Zentgraf, T.; Ma, R.-M.; Gladden, C.; Dai, L.; Bartal, G; Zhang, X. Plasmon lasers at deep subwavelength scale. Nature 2009, 461, 629.

(8) Saxena, D.; Mokkapati, S.; Parkinson, P.; Jiang, N.; Gao, Q.; Tan, H.H.; Jagadish, C. Optically pumped room-temperature GaAs nanowire lasers. Nature Photon. 2013, 7, 963.

(9) Marcinkevičius, S.; Leon, R. Carrier capture and escape in $\operatorname{In}_{x} \mathrm{Ga}_{1-\mathrm{x}} \mathrm{As} / \mathrm{GaAs}$ quantum dots: Effects of intermixing. Phys. Rev. B 1999, 59, 4630.

(10) Tatebayashi, J.; Kako, S.; Ho, J.; Ota, Y.; Iwamoto, S.; Arakawa, Y. Roomtemperature lasing in a single nanowire with quantum dots, Nature Photonics 2015, 9, 501. 